\title{
Analysis of Particle Size Distributions of Quantum Dots: From Theory to Application ${ }^{\dagger}$
}

\author{
Doris Segets \\ ${ }^{1}$ Institute of Particle Technology, Friedrich-Alexander-Universität Erlangen-Nürnberg (FAU), Germany
}

\begin{abstract}
Small, quantum-confined semiconductor nanoparticles, known as quantum dots (QDs) are highly important material systems due to their unique optoelectronic properties and their pronounced structure-property relationships. QD applications are seen in the emerging fields of thin films and solar cells. In this review, different characterization techniques for particle size distributions (PSDs) will be summarized with special emphasis on strategies developed and suggested in the past to derive data on the dispersity of a sample from optical absorbance spectra. The latter use the assumption of superimposed individual optical contributions according to the relative abundance of different sizes of a colloidal dispersion. In the second part, the high potential of detailed PSD analysis to get deeper insights of typical QD processes such as classification by size selective precipitation (SSP) will be demonstrated. This is expected to lead to an improved understanding of colloidal surface properties which is of major importance for the development of assumption-free interaction models.
\end{abstract}

Keywords: particle size distribution, quantum dot, structure-property relationship, classification by size selective precipitation, solubility parameters

\section{Introduction}

Quantum dots (QDs) are semiconductor nanoparticles which are small enough that quantum confinement occurs in all three dimensions. The first systematic dynamic investigations of interfacial electron and hole processes as well as reports on the size-dependent optical properties of QDs in solution date back to the early 1980s (Duonghong D. et al., 1982; Kuczinski J. and Thomas J.K., 1982; Rossetti R. et al., 1984). Therefrom motivated, Brus was the first who developed a quantum mechanical model based on the effective mass approximation (EMA) of electrons and wholes to predict the evolution of the size-dependent band gap energy (Brus L.E., 1983, 1984; Steigerwald M.L. and Brus L.E., 1990).

Afterwards, as recently summarized by Tyrakowski and Snee (Tyrakowski C.M. and Snee P.T., 2014), more than two decades of intense research on QDs passed, covering various aspects from quantum mechanics and optical properties (Alivisatos A.P., 1996), particle synthesis (Park J. et al., 2007), functionalization and ligand exchange (Alvarado S.R. et al., 2014; Lin W. et al., 2015).

Received 15 May 2015; Accepted 26 June 2015

J-STAGE Advance published online 10 September 2015

Cauerstraße 4, 91058 Erlangen, Germany

E-mail: Doris.Segets@fau.de

TEL: +49-9131-85-29404 FAX: +49-9131-85-29402
Nowadays-although not yet an established mass product-QDs are commercially available via online shops and are highly relevant for the emerging fields of printed solar cells (Debnath R. et al., 2011) and thin-film electronics (Choi J.-H. et al., 2012; Moynihan T., 2015). Moreover, they become increasingly attractive for medical and biological applications (Cottingham K., 2005).

The fine-tuning of a PSD is decisive for any kind of later application. This is due to the aforementioned pronounced size-property relationships of those transient structures situated at the interface between dissolved molecules and solid particles. Thus, QDs are inherently related to interdisciplinary research. Whereas the molecular, rather than the chemical and physical approach is the point where investigations of QDs have originally been started, those nanoparticles provide many aspects that are of equal interest to the field of process engineering and particle technology. Usually, QDs with tailored optical properties in terms of defined absorption onset and emission features in combination with a narrow size distribution are required (Nightingale A.M. and De Mello J.C., 2010; Yen B.K. et al., 2005). This is not only a central issue related to scale-up but equally demanding in terms of characterization-especially when keeping in mind the small particle sizes of a few nanometers in maximum.

To quantitatively compare the narrowness of a PSD throughout different samples, the relative standard deviation (RSD) or coefficient of variation, defined as the stan- 
dard deviation divided by the mean particle size $\left(\mathrm{RSD}=\sigma / x_{\text {mean }}\right)$, is usually applied. Following the convention, "monodispersity" is achieved if RSD $<0.05$, whereas "narrow" PSDs are found for RSD $<0.1$. This fundamental requirement of narrow distributions raises the following central questions:

i) How can QDs that cover particle sizes clearly below $10 \mathrm{~nm}$, often even down to $1 \mathrm{~nm}$, be accurately characterized with justifiable experimental effort?

ii) To what extent can narrow PSDs with RSDs below 0.1 or even below 0.05 be achieved?

iii) Can knowledge on i) and ii) be used to access particleparticle interactions of small colloids?

Whereas the second issue has already recently been discussed by Kowalczyk and Mori and the second issue at least partly addressed by Mori (Kowalczyk B. et al., 2011; Mori Y., 2015), the challenge of size characterization remains. Therefore, in the following sections, different strategies based on the deconvolution of absorbance measurements developed by different groups will be discussed and compared to standard and advanced methods of small particle characterization found in the field of QDs and/or particle technology. The latter will be done with respect to accuracy, availability, experimental effort and potential of the method to become a suitable online technique. In the last part of this work, the high potential of size analysis - aiming to make particle interactions accessible — will be demonstrated by a more recent example on QD classification by size selective precipitation (SSP) (Segets D. et al., 2015). The study shows how careful particle characterization on an $\AA$-level is of paramount importance in order to access colloidal surface properties, and how those may be linked in the near future to permit calculation of assumption-free interaction potentials.

\section{Size characterization of QDs}

In the following sections, methods for QD size characterization will be introduced. First, standard methods such as (high-resolution (HR)) transmission electron microscopy (TEM) and dynamic light scattering (DLS) as well as advanced techniques such as small-angle X-ray scattering (SAXS), field-flow fractionation (FFF) and analytical ultracentrifugation (AUC) will be discussed briefly. Then, special emphasis will be placed on different approaches for the deconvolution of absorption data to derive a PSD beyond a mean particle size and a standard deviation.

\section{(High-resolution) transmission electron microscopy}

At first glance, the most obvious way to analyze QDs is (HR)TEM. It not only gives access to the primary particle size and shape - most of QDs are in good approximation spherical, however, other shapes such as cubes are reported as well (Pietryga J.M. et al., 2004) — but also allows a discrimination of isolated particles from aggregates or superstructures. Moreover, crystallinity and thus the inner structure of the particles can be derived directly from TEM data. Drawbacks are the necessity of an additional drying step and poor statistics that need to be carefully considered when conclusions based on a limited amount of particles $(<1000)$ are to be drawn for the ensemble $\left(>>10^{10}\right)$. Finally, side effects such as ripening on the TEM substrate induced by the electron beam need to be considered as well (Segets D. et al., 2013). Although over the past years, reports on liquid cell TEM (Nielsen M.H. et al., 2014; Zheng H. et al., 2009) and the evaluation of larger datasets due to automatized image analysis (Segets D. et al., 2012; Sun Y. et al., 2012) came more and more into the focus of interest, electron microscopy is demanding and not (yet) suitable for larger sample numbers and online analysis.

\section{Dynamic light scattering}

A frequently used technique for particle size analysis is DLS. It is commercially available, applicable in the liquid phase, and comparatively fast ( $\sim$ min). It is based on the dynamic change in the scattering of coherent, monochromatic laser light due to the diffusion of small particles in a fluid. An autocorrelation function for the analysis of those fluctuations gives access to the diffusion coefficient $D$. With known temperature $T$ and viscosity of the fluid $\eta$, the hydrodynamic particle diameter $x_{\mathrm{h}}$ (particle core + adsorbate/ligand shell) is available via the Stokes-Einstein equation:

$$
D=\frac{k_{\mathrm{B}} T}{3 \pi \eta x_{\mathrm{h}}}
$$

If the extinction-weighted distribution of hydrodynamic particle sizes is to be transferred to a volume or number PSD, a Mie correction is necessary to consider the size-dependent extinction coefficient.

Regarding smallest nanoparticles such as QDs, it becomes obvious that a core size distribution is hardly accessible via DLS due to the fact that the ligand shell is no longer negligible (Marczak R. et al., 2010; Oshima H., 2009; Reindl A. and Peukert W., 2008). Additionally, the scattering intensity of particles below $10 \mathrm{~nm}$ is small, causing a low resolution of commercial devices in the size range of interest between 1 and $10 \mathrm{~nm}$.

\section{Small-Angle X-ray Scattering}

In addition to the already mentioned standard techniques, other methods also exist to characterize PSDs with good or even outstanding accuracy. For instance, SAXS can be applied in situ to characterize colloids with 
respectio primary particle size and, if required, gives accass to agglomerates and even hierarchical structures such as mesocrystals (Seto J. et al., 2012; Sun Y. and Ren Y., 2013). The scattering signal correlates with the electron density distribution within the sample and is thus sensitive to concentration, size and shape (Keshari A.K. and Pandey A.C., 2008; Pauw B.R., 2013). As only one superimposed scattering curve is recorded, data interpretation is challenging and cross-validation against other, independent techniques is required. However, once established for a certain system, SAXS is a powerful technique for particle analysis (Abécassis B. et al., 2015; Caetano B.L. et al., 2010; Polte J. et al., 2010).

\section{Field-Flow Fractionation}

A versatile method for the size characterization of not only QDs but also of proteins, polymers, macromolecules, cells and colloids in general is field-flow fractionation (FFF) (Baalousha M. et al., 2011). It is a chromatographic technique, which means that it includes classification of the solute during the measurement. This is seen as a clear advantage with respect to resolution and also with respect to detection (Kowalczyk B. et al., 2011; Mori Y., 1994, 2015). Regarding the measurement principle, no column is needed but separation rather occurs in a laminar flow channel which is superimposed with an orthogonal second field. This second field is often a flow field, but also thermal, electric, magnetic and gravitational fields are possible (Baalousha M. et al., 2011; Williams S.K.R. et al., 2011). For nanoparticles, asymmetric flow FFF (AsFlFFF or A4F) is usually applied. A further advantage of FFF is the flexible detection that can be realized online (e.g. UV/ Vis, organic carbon, fluorescence) or offline (e.g. TEM, atomic force microscopy, AUC). Thus, although the investment costs are comparatively high and solute concentrations are an issue, FFF is a promising technique, especially in combination with multiple detectors and with other, complementary analytical methods for size characterization (Dieckmann Y. et al., 2009; Hagendorfer H. et al., 2012).

\section{Analytical Ultracentrifugation}

Another outstanding characterization technique is AUC. Due to rotor speeds of up to $60 \mathrm{krpm}$, it has unrivaled resolution on an $\AA$-level and can detect smallest particles even below $1 \mathrm{~nm}$. The sedimentation of the particles in a centrifugal field is recorded and - usually based on extinction analysis over time-the sedimentation velocity is derived. From this, the particle size, shape and surface properties in terms of the ligand shell are accessible, which makes AUC an excellent technique for the characterization of smallest colloids (Lees E.E. et al., 2008). In parallel, special emphasis has been devoted to the development of advanced evaluation routines and al- gorithms (Brookes E.H. and Demeler B., 2008; Walter J. et al., 2015b). More recently, AUC has been developed further to allow not only for the detection of a single wavelength but to extend for multiwavelength (MWL) data analysis (Balbo A. et al., 2005; Bhattacharyya S.K. et al., 2006; Strauss H.M. et al., 2008; Walter J. et al., 2014). As a result, the optical properties of individual species in a mixture, such as the absorbance of discrete-sized QDs, will become accessible.

Although the availability of AUC is still limited and online analysis is not possible, it is expected to become the gold standard for particle characterization due to its unmatched resolution of multimodal size distributions and the possibility to extend towards multidimensional characterization. For instance, size and spectral properties, size and shape or size and density (Carney R.P. et al., 2011; Walter J. et al., 2014; Walter J. et al., 2015a; Walter J. et al., 2015b).

At this point it should be briefly mentioned that the same holds true for analytical centrifugation (AC) which works with lower gravitational fields but extends the measurement range to larger particle sizes (Detloff T. and Lerche D., 2008; Jafarzadeh S. et al., 2011; Krause B. et al., 2010; Walter J. et al., 2015c). Noteworthy, offline information from AUC, like for instance the optical properties of discrete QD fractions, could be implemented in evaluation routines based on UV/Vis spectroscopy. The latter is widely available and well-suited for fast online investigations which are needed for kinetic studies at high-time resolution ( $\sim \mathrm{ms})$. Hence, the technique will be discussed in more detail in the following text with special emphasis on different approaches of data evaluation.

\subsection{Deconvolution of absorbance spectra}

A rather simple and straightforward strategy for the inline PSD analysis of QDs is the use of optical properties such as absorption and emission. This is possible due to the pronounced structure-property relationships of these materials such as-one of the most prominent examples - the size-dependent band gap energy $E_{g}(x)$. As exemplarily depicted in Fig. 1 for Cd-based materials, both absorbance and emission can be tuned over a wide wavelength range by adjusting the particle size (Yu W.W. et al., 2003).

Knowledge of $E_{\mathrm{g}}(x)$ allows correlation of different features of an absorbance spectrum such as i) wavelength of the absorption onset, ii) wavelength of the absorption maximum of the first peak or iii) point of inflexion with discrete particle diameters. This leads to a rough estimation of the particle sizes present in a sample. However, although QD size distributions are narrow, often with RSDs below 0.1 or even below 0.05 , Mićić et al. pointed out more than 20 years ago that already small polydispersity 


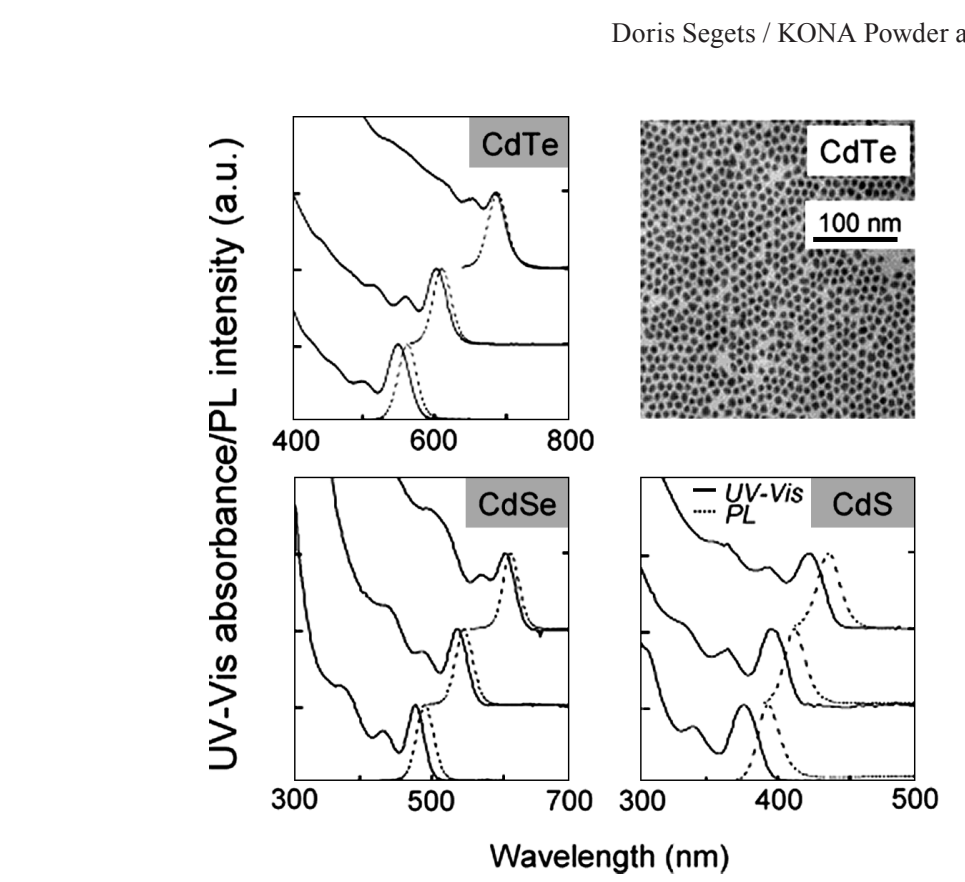

Fig. 1 UV/Vis and PL spectra of CdTe, CdSe and CdS nanocrystal samples. A typical TEM image of CdTe is shown in the upper right corner. Reprinted with permission from Yu et al., "Experimental Determination of the Extinction Coefficient of CdTe, CdSe, and CdS Nanocrystals" Chem. Mater. 2003 15(14), p. 28542860. Copyright 2003 American Chemical Society.

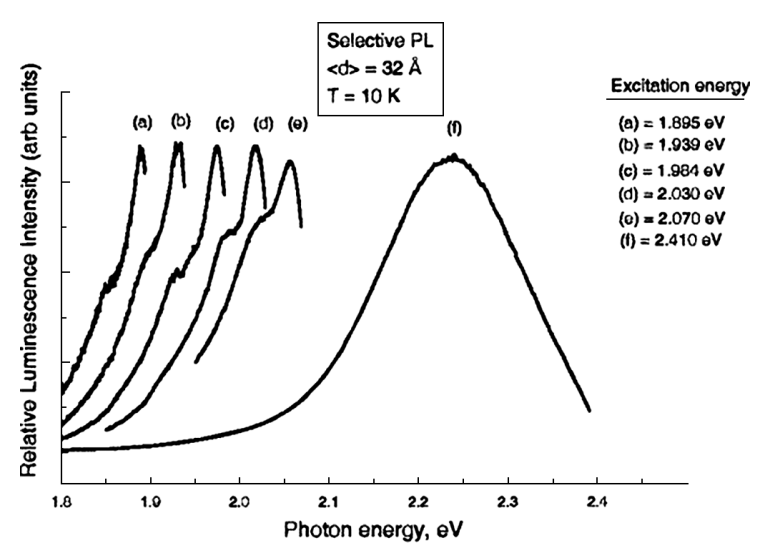

Fig. 2 PL spectra at $10 \mathrm{~K}$ for an ensemble of InP quantum dots with a mean diameter of $3.2 \mathrm{~nm}$ for different excitation energies. The first absorption peak of the QD ensemble is at $2.17 \mathrm{eV}$, so that the PL curves a-e result from excitation $(1.895-2.070 \mathrm{eV})$ in the red tail of the onset region of the absorption spectrum and are fluorescence-line-narrowing (FLN) spectra; curve $\mathrm{f}$ is a global PL spectrum since its excitation was at $2.14 \mathrm{eV}$ and is well into the blue end of the first absorption peak. Reprinted with permission from Mićić et al., "Size-Dependent Spectroscopy of InP Quantum Dots" J. Phys. Chem. B 1997 101(25), p. 4904-4912. Copyright 1997 American Chemical Society.

possibly not as accurate as SAXS, FFF and/or AUC, UV/ Vis absorbance spectroscopy is a standard technology that is widely available and suitable for fast inline analysis $(\sim \mathrm{ms})$.

At this point it needs to be mentioned that photoluminescence (PL) spectroscopy is sometimes applied as well to get an impression of the dispersity of a sample (Peng X. et al., 1998). However, as already mentioned by the authors of this work, the assumptions of (i) a $\delta$-function emission and (ii) a size-independent emission intensity lead to an overestimation of the width of the reported PSDs. Moreover, as later pointed out by Viswanatha and Sarma, the PL approach is restricted to fully passivated samples and thus would, e.g. fail in the case of $\mathrm{ZnO}$ QDs that do not show a band edge tuning (Viswanatha R. and Sarma D.D., 2006).

Therefore in the following text, only approaches based on absorbance analysis will be discussed (Mićić O.I. et al., 1994; Pesika N.S. et al., 2003a; Pesika N.S. et al., 2003b; Segets D. et al., 2009; Segets D. et al., 2012; Viswanatha R. and Sarma D.D., 2006). Despite differences in the exact deconvolution procedure, they all use the same basic assumptions and restrictions, namely

i) semiconductor nanoparticles with a direct band gap which are small enough that the scattering contribution to the total extinction is negligible and quantum confinement occurs,

ii) a monotone dependency between particle size and 
nis

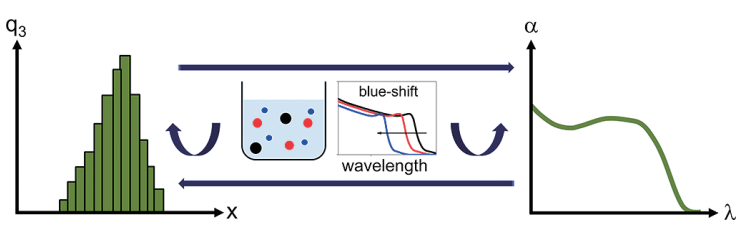

Fig. 3 Sketch of the correlation between polydispersity in particle size and absorbance that is finally accessible by UV/Vis/NIR analysis (Segets D. and Peukert W., 2014).

band gap energy,

iii) a linear superposition of the individual contributions of the different particle size fractions as illustrated in Fig. 3.

Within a polydisperse sample of quantum-confined particles, every size fraction has its specific absorption spectrum. According to the relative abundance of the differently sized QDs in the macroscopic sample, the global absorption is created. The challenge is to deduce from an absorbance measurement the size of the underlying species together with their relative abundance and therefrom to create a PSD.

Approach 1: Least squares fit and pre-assumption of PSD shape

Mićić et al. were the first who derived PSDs from absorbance data. In doing so, they already followed a highly innovative two-step approach (Mićić O.I. et al., 1994). First, they synthesized rather polydisperse InP QDs and determined the underlying PSD by HRTEM. Then, they derived by a fit to experimental data (Palik E.D., 1985) an analytical equation for the absorption coefficient $\alpha$ as a function of energy $h v$ (or wavelength, respectively) and band gap $E_{\mathrm{g}}$. The latter included the particle size $x$ (or strictly the particle radius $r=x / 2$ ) and two fitting parameters $n$ and $p$ :

$$
\alpha=f\left[h v, E_{\mathrm{g}}(r, n, p)\right]
$$

Now assuming a PSD following the frequently observed shape of a lognormal distribution, a least squares fit was performed to find a PSD that gives a best match with the measured absorption data (Fig. 4, dataset a). Noteworthy is that after having calibrated parameters $n$ and $p$, they were kept constant throughout all further PSD calculations that matched the expectations well (Fig. 4, datasets b and c) (Mićić O.I. et al., 1994).

Disadvantages are the limited number of particles that were evaluated for the calibration PSD, the missing validation of $E_{\mathrm{g}}(x)$ against literature data and the predefined shape of the PSD. However, the first two points can be clearly attributed to the time when the study was performed. In the early 90 s, TEM capabilities were much lower than nowadays and reference data did not yet exist

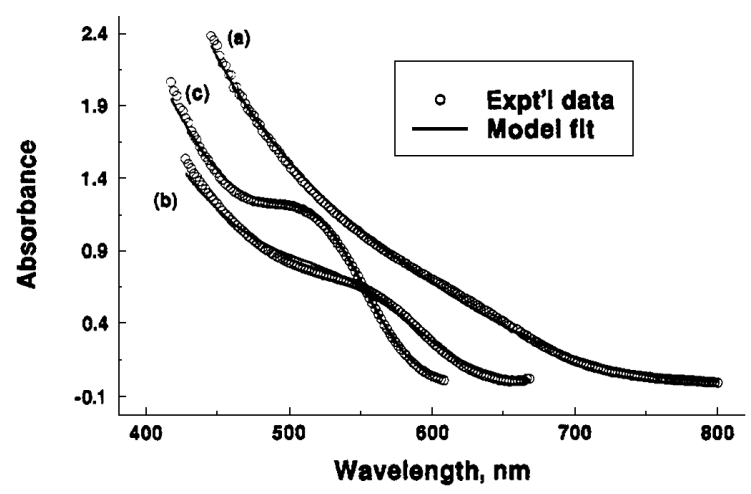

Fig. 4 Experimental absorption spectra (circles) for InP QD colloids with fit to model shown as a solid line: (a) fit made to yield the dependence of QD band gap on QD size; ( $b$ and c) fit made to yield the size distribution from the results of (a). Reprinted with permission from Mićić et al., "Synthesis and Characterization of InP Quantum Dots" J. Phys. Chem. 1994 98(19), p. 49664969. Copyright 1994 American Chemical Society.

as QDs and related research had just started to come into being. Thus, the work is pioneering in a way that it not only allows the calculation of non-symmetric lognormal PSDs, but even offers the possibility to derive $E_{\mathrm{g}}(x)$ as a material property.

More than a decade later, Viswanatha and Sarma confirmed the applicability of this concept to other material systems by generalizing it to experimental data of $\mathrm{ZnO}$, $\mathrm{ZnS}$ and CdSe QDs (Viswanatha R. and Sarma D.D., 2006). In contrast to the earlier work of Mićić, they used Gaussian instead of lognormal distributions and did not calibrate $E_{\mathrm{g}}(x)$ but used fundamental literature data. For instance, in the case of $\mathrm{ZnO}$, a carefully validated tight-binding model (TBM) was applied (Viswanatha R. et al., 2004). Moreover, they proved the assumption of the parallel-shifted bulk absorption spectrum to higher energies for decreasing particle sizes. Therefore they compared the results of the original approach to the outcome when a simple 0-1 step function was used for the shape of the absorption coefficient $\alpha$. It was found that the difference in the calculated PSDs was negligible. Finally, the apparent width of the experimental and simulated absorbance data of various material systems $(\mathrm{ZnO}, \mathrm{ZnS}(\mathrm{e})$, $\mathrm{CdS}(\mathrm{e}), \mathrm{GaAs}, \mathrm{InAs}, \mathrm{InP})$ on a particle size scale $\left(\Delta d_{\mathrm{app}}\right)$ was compared to the true widths of the underlying PSDs $\left(\Delta D_{\text {actual }}\right)$. A material-independent, empiric relationship was thereby identified that allows a quick estimate of a sample's polydispersity from $\Delta d_{\text {app. }}$. In addition to the absorption data, only knowledge of $E_{\mathrm{g}}(x)$ is required:

$$
\Delta D_{\text {actual }}=-0.0025 \times \frac{\Delta d_{\mathrm{app}}^{2}}{n m}+0.524 \times \Delta d_{\mathrm{app}}-1.41 \mathrm{~nm}
$$

However, for both approaches, the aforementioned is- 


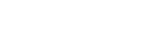

sue of $a^{\circ}$ predefined shape of the underlying PSD remains. Thierefore, in the following sections, two alternatives that are able to access multimodal distributions without any assumptions on the PSD shape(s) will be discussed.

Approach 2: Wavelength-based deconvolution for arbitrary shaped PSDs

In 2003, the first concept of deriving arbitrary shaped PSDs from absorbance data was developed by Pesika et al. (Pesika N.S. et al., 2003a; Pesika N.S. et al., 2003b). Although the first report of their method was rather restricted to $\mathrm{ZnO}$ QDs, they summarized the procedure in the same year again with a more general point of view.

Based on absorbance data of a (0001) $\mathrm{ZnO}$ single crystal (Fig. 5), they came up with a comparatively simple but useful assumption, namely that in the case of a direct band gap semiconductor, the absorption coefficient $\alpha$ in the vicinity of the onset is given by (Pesika N.S. et al., 2003a; Pesika N.S. et al., 2003b):

$$
\alpha=\frac{C\left(h v-E_{\mathrm{g}}^{\mathrm{bulk}}\right)^{1 / 2}}{h v}
$$

$C$ is a constant, $h v$ is the photon energy and $E_{\mathrm{g}}$ bulk is the bulk band gap.

Using an effective mass model according to Brus (Brus L., 1986) for the correlation between band gap energy and particle size and further assuming spherical particles and a size-independent absorption coefficient, the following connection between absorbance $A$, particle size $x$ and relative abundance $n$ was derived (Pesika N.S. et al.,

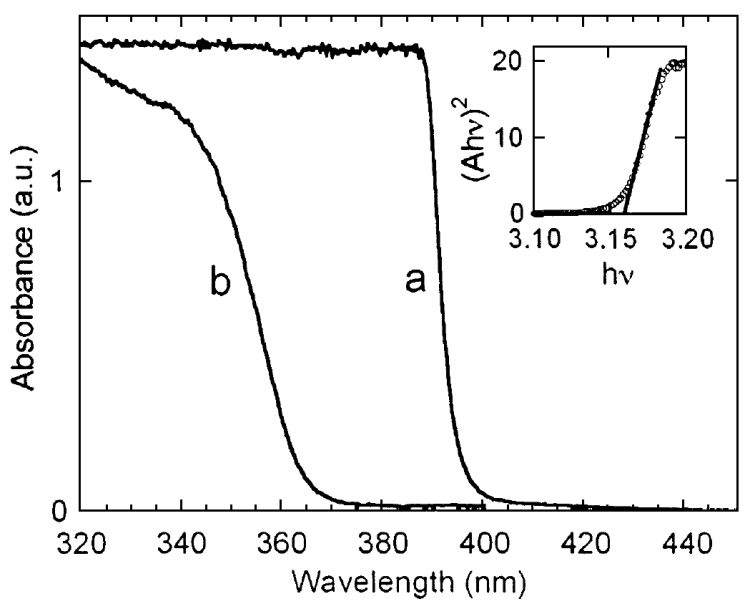

Fig. 5 (a) Absorbance spectrum for a (0001) $\mathrm{ZnO}$ single crystal and (b) absorbance spectrum for a suspension of $\mathrm{ZnO}$ quantum particles after $2 \mathrm{~h}$ of growth at $65^{\circ} \mathrm{C}$. The inset shows the spectrum for the $\mathrm{ZnO}$ single crystal plotted as $(A h v)^{2}$ versus $h v$. Reprinted with permission from Pesika et al., "Relationship between Absorbance Spectra and Particle Size Distributions for Quantum-Sized Nanocrystals" J. Phys. Chem. B 2003 107(38), p. 10412-10415. Copyright 2003 American Chemical Society. 2003a; Pesika N.S. et al., 2003b):

$$
A(x) \propto \int_{x}^{\infty} \frac{\pi}{6} x^{3} n(x) \mathrm{d} x
$$

From Eq. 5 it becomes clear that (i) the absorbance of QDs is a volume signal that (ii) can be used to calculate the relative abundance of distinct sizes from the local slope of the absorbance spectrum (boundary condition: $n(x)=0$ for $x \rightarrow \infty)$ :

$$
n(x) \propto-\frac{\frac{\mathrm{d} A}{\mathrm{~d} x}}{\frac{\pi}{6} x^{3}}
$$

As becomes clear from Fig. 6, validation of the calculation results revealed an excellent agreement with the size distributions determined by TEM. However, in contrast to the formerly discussed approaches, even bimodal distributions of arbitrary shape could be derived without any input parameters. This was proven by the evaluation of absorbance data of mixed suspensions containing controlled amounts of small and large particles. A nearly perfect match was found when the results from the bimodal absorbance spectrum were compared to the expected distributions obtained from the individually evaluated absorption data of small and large particles. Another advantage of the technique is its comparatively simple implementation as only a correlation for the size-dependent band gap energy $E_{\mathrm{g}}(x)$ and the local slope of the absorbance spectrum are required. The only drawback, how-

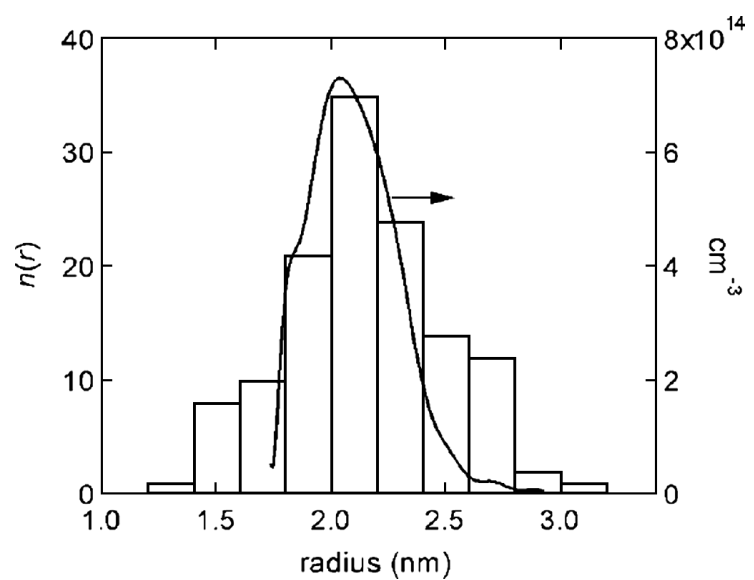

Fig. 6 Distribution results of $\mathrm{ZnO}$ QDs after $2 \mathrm{~h}$ of growth at $65^{\circ} \mathrm{C}$. The histogram was obtained from analysis of high-resolution transmission electron microscope images of 125 particles. The solid line was obtained from the absorbance spectrum. Reprinted with permission from Pesika et al., "Relationship between Absorbance Spectra and Particle Size Distributions for QuantumSized Nanocrystals" J. Phys. Chem. B 2003 107(38), p. 10412-10415. Copyright 2003 American Chemical Society. 
gider, is the strong assumption of a step function for the absorbance of monodisperse particles. Simply trying to make a reconstruction of the original absorbance spectrum by using a known $n(x)$ and applying Eq. 5, the minimum observed after the first absorbance peak is never obtained-especially in the case of smaller nanoparticles.

To address this issue, Segets et al. developed a more flexible approach with respect to the shape evolution of the shifted absorption coefficient (Segets D. et al., 2009; Segets D. et al., 2012). Instead of using a step function, the bulk absorption coefficient was used as an assumption for the shape of the monodisperse QD absorption evolution. The size dependency was simply addressed by a parallel shift of the bulk absorption to smaller wavelengths according to $E_{\mathrm{g}}(x)$. Regarding the deconvolution of a measured absorbance spectrum $A(\lambda)$ into partial spectra $A_{i}{ }^{\prime}(\lambda)$, each of these assumed to have the form of the bulk absorption $\alpha(\lambda)$, the algorithm is realized as follows (Segets D. et al., 2009; Segets D. et al., 2012):

(i) First, the bulk absorption $\alpha$ is parallel-shifted in a way that its peak position matches the largest wavelength under consideration $\left(\lambda_{i}\right)$. The particle diameter that is related to this specific wavelength needs to be derived either from quantum mechanics or from a separate calibration by matching calculated PSDs ${ }^{\text {calc }}$ against PSDs ${ }^{\text {TEM }}$, as suggested by Mićic et al. In the following text, this shifted absorption coefficient will be denoted as $\alpha_{\text {shift } i}(\lambda)$.

(ii) Then, $\alpha_{\text {shift }, i}(\lambda)$ is scaled in a way that it coincides with the measurement value $A\left(\lambda_{i}\right)$ :

$$
A_{i}{ }^{\prime}(\lambda)=\frac{\alpha_{\mathrm{shift}, i}(\lambda)}{\alpha_{\mathrm{shift}, i}\left(\lambda_{i}\right)} \times A_{i}\left(\lambda_{i}\right)
$$

This leads to the first partial absorbance spectrum that exactly matches the measurement value at position $\lambda_{i}$.

(iii) Dividing $A_{i}\left(\lambda_{i}\right)$ by the value of the shifted absorption coefficient at this wavelength $\left(\alpha_{\text {shift,i }}\left(\lambda_{\mathrm{i}}\right)\right)$, the particle size interval $\Delta x=x_{i+1}-x_{i}$ under consideration and the optical path length $d_{\mathrm{c}}$, the first value of a size distribution that is still related to the total mass of solid QD material is obtained (Segets D. et al., 2009). Worthy of note is that $x_{i+1}$ is the particle size which is linked with the next smaller wavelength analyzed. Thus, $\Delta x_{i}$ depends on the measurement settings.

(iv) Then, the absorbance contribution of this known, largest particle size fraction is subtracted from the measurement spectrum according to:

$$
A(\lambda)=A(\lambda)-A_{i}{ }^{\prime}(\lambda)
$$

Thus, a new spectrum is created and steps (i-iv) are repeated until all relevant wavelengths are analyzed.

(v) Finally, the concentration-related distribution is converted to a PSD that fulfills the requirement of a nor-
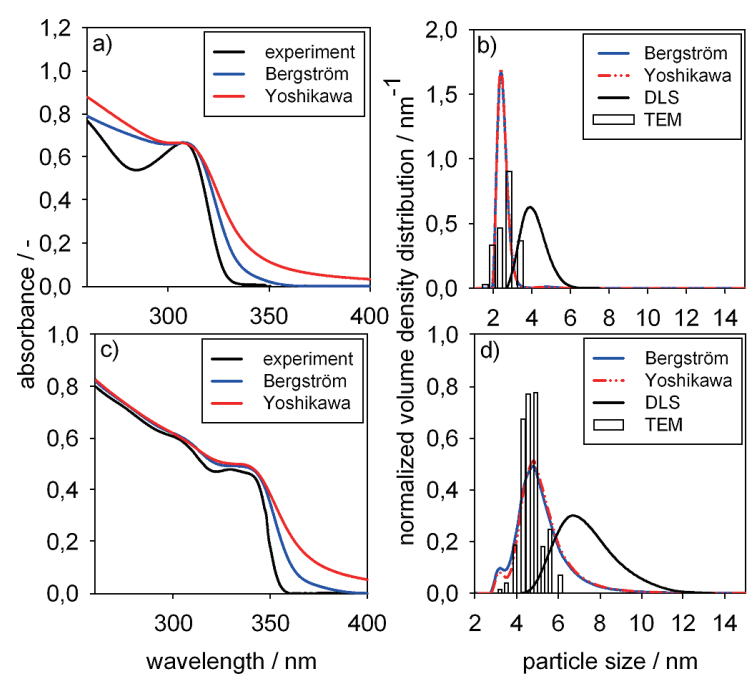

Fig. 7 Comparison between the calculated PSDs, DLS and optical image analysis of two different samples of b) small and d) large $\mathrm{ZnO}$ QDs and the related measured (black solid line) and reconstructed (blue and red solid lines) absorbance spectra for a) smaller and c) largersized samples. Reprinted with permission from Segets et al., "Analysis of Optical Absorbance Spectra for the Determination of $\mathrm{ZnO}$ Nanoparticle Size Distribution, Solubility, and Surface Energy" ACS Nano 2009 3(7), p. 1703-1710. Copyright 2009 American Chemical Society.

malized density distribution:

$$
\sum_{x_{\min }}^{x_{\max }}\left[q_{3}(x) \cdot \Delta x\right]=1
$$

As already expected from the analysis of Viswanatha and Sarma (Viswanatha R. and Sarma D.D., 2006) using Gaussian distributions, the final results are not strongly affected by the assumed shape of the parallel-shifted bulk absorption (Segets D. et al., 2012) also in the case of arbitrary PSDs. To illustrate this, Fig. 7 shows the deconvolution results of absorbance data derived for small $(a, b)$ and large (c, d) ZnO QDs using two different datasets for the bulk absorption coefficient (Bergström L. et al., 1996; Yoshikawa H. and Adachi S., 1997). For validation, TEM and DLS results are presented as well.

It becomes clear that while the calculated PSDs are not affected by the dataset used for the absorption coefficient, the reconstructed absorbance measurements do depend on the optical properties chosen. Noteworthy is that the shifted bulk absorption is not yet expected to be the correct absorption evolution of monodisperse QDs. However, as soon as discrete optical properties are accessible, for instance via AUC analysis, they are easily implemented to the existing code. A proof of the correct optical properties would be obtained as soon as not only the forward calculation of a PSD ${ }^{\text {calc }}$ is done correctly, but when also 


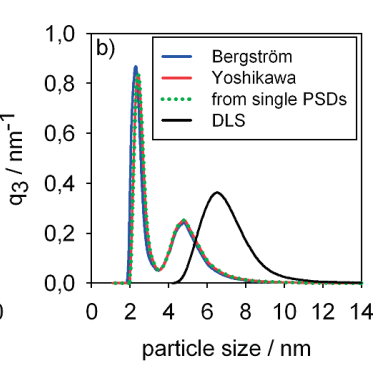

Fig. 8 a) Absorbance spectrum of the bimodal PSD after mixing the two suspensions in the same volume ratio (black line) and its corresponding reconstructions (blue and red lines); b) bimodal PSD calculated from the bimodal absorbance spectrum with the bulk properties from Bergström and Yoshikawa (blue and red lines), expected bimodal PSD calculated from the PSDs of the pure unmixed suspensions (green dotted line), and bimodal PSD measured with DLS (black line). Reprinted with permission from Segets et al., "Analysis of Optical Absorbance Spectra for the Determination of $\mathrm{ZnO}$ Nanoparticle Size Distribution, Solubility, and Surface Energy" ACS Nano 2009 3(7), p. 1703-1710. Copyright 2009 American Chemical Society.

the reconstructed absorbance spectrum based on $\mathrm{PSD}^{\text {calc }}$ matches the measurement data. As already discussed, the analysis of bimodal distributions without any input parameters is possible as well using this approach. This is summarized in Fig. 8.

Additionally, in a subsequent work, the original idea of Mićić to derive $E_{\mathrm{g}}(x)$ by careful calibration was picked up again (Segets D. et al., 2012). A study on $\mathrm{PbS}(\mathrm{e})$ was performed to demonstrate the general applicability of the method originally developed for $\mathrm{ZnO}$ by means of a completely different QD system. In addition, in the case of $\mathrm{PbS}(\mathrm{e})$ QDs that were chosen for this study, well-established and properly validated literature data on $E_{\mathrm{g}}(x)$ is available. Small and large particles were synthesized and aberration-corrected TEM micrographs were analyzed by semi-automatized image analysis. In parallel, a power law with three empiric parameters $a[\mathrm{~nm}], b[-]$ and $c[\mathrm{~nm}]$ was used for description of the size-dependent band gap energy $\Delta E_{i}\left(x_{i}\right)$ :

$$
x_{i}=a\left(\frac{\Delta E_{i}}{e V}\right)^{b}+c
$$

Finally, $a, b$ and $c$ were modified until a best match was obtained between PSDs ${ }^{\text {calc }}$ derived by the algorithm and $\mathrm{PSDs}^{\mathrm{TEM}}$ derived by image analysis. It was found to be preferable to analyze two samples covering two different particle size regimes in order to arrive at compromise values for $a, b$ and $c$. This leads to a function of $E_{\mathrm{g}}(x)$ which is valid over a wide size range. The results are shown in Fig. 9.
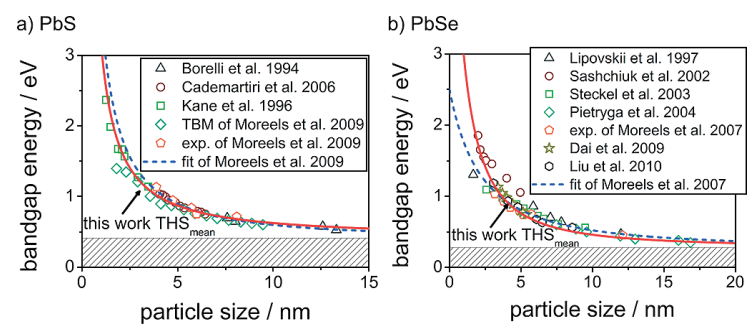

Fig. 9 Relationship between fundamental band gap energy and particle size as derived in this work along with comparison to literature data for a) $\mathrm{PbS}$ (Borrelli N.F. and Smith D.W., 1994; Cademartiri L. et al., 2006; Kane R.S. et al., 1996; Moreels I. et al., 2009) and b) PbSe (Dai Q. et al., 2009; Koole R. et al., 2008; Lipovskii A. et al., 1997; Liu Y. et al., 2010; Ma W. et al., 2011; Moreels I. et al., 2007; Pietryga J.M. et al., 2004; Sashchiuk A. et al., 2001; Steckel J.S. et al., 2003) as indicated. Reprinted with permission from Segets et al., "Determination of the Quantum Dot Band Gap Dependence on Particle Size from Optical Absorbance and Transmission Electron Microscopy Measurements" ACS Nano 2012 6(10), p. 9021-9032. Copyright 2012 American Chemical Society.

It becomes clear that an excellent agreement exists with literature data collected over the past decades. This confirms the large potential of QD absorbance data analysis. Thus, the usefulness of PSD calculation from absorbance measurements by various approaches with all of them having their specific advantages and disadvantages is sufficiently proven. Future directions are seen in the combination of different characterization methods (e.g. SAXS, FFF, AUC and UV/Vis analysis) to increase accuracy and resolution even further.

Especially the latter is somewhat limited by the absorbance approach as i) information on small particles might be hidden in the tail of absorbance spectra of larger sizes and as ii) the resolution is somewhat limited by the requirement of a noticeable change of $E_{\mathrm{g}}(x)$ for two different particle diameters. Therefore, AUC that provides a maximum knowledge gain with respect to a PSD with sub-nm resolution, composition and surface chemistry becomes one of the most important techniques for QD characterization (Carney R.P. et al., 2011; Lees E.E. et al., 2008). In combination with in-situ techniques such as UV/Vis or SAXS, it is expected to provide new, mechanistic insights of QD formation with unrivaled accuracy.

\section{Application to classification}

At this stage, some fundamental conclusions with respect to colloidal interactions are possible. This is demonstrated by applying the method to classification on a sub-10 nm scale. Worthy of note is that various approaches 


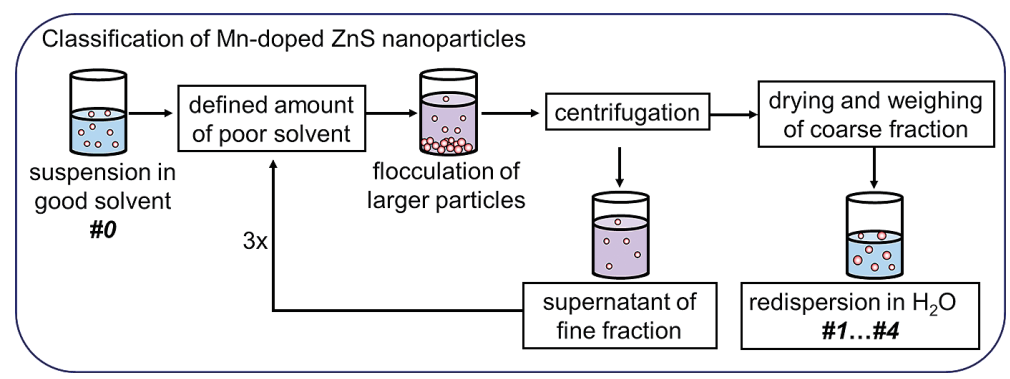

Fig. 10 Illustration of the experimental procedure of SSP. With kind permission from Springer Science+Business Media: Segets et al., "Quantitative evaluation of size selective precipitation of Mn-doped ZnS quantum dots by size distributions calculated from UV/Vis absorbance spectra" J. Nanopart. Res. 2013 15:1486, Scheme 1.

for nanoparticle classification and purification exist that are already described in excellent review papers (Kowalczyk B. et al., 2011; Mori Y., 2015; Sapsford K.E. et al., 2011). However, for QDs, size selective precipitation (SSP) which is the preferred flocculation of larger particles in a non-solvent-is still the most important post-processing strategy.

In the following sections, first the working principle of SSP will be introduced. It will be discussed how SSP can be described theoretically based on results obtained from careful PSD and mass balance analysis. Finally, things are set into perspective by an outlook that shows how classification by SSP is linked to Hansen solubility parameters (HSP) which in turn give access to the Flory-Huggins interaction parameter $\chi$. Knowledge of $\chi$ is decisive for derivation of the osmotic interactions between the ligand shells around QDs and thus represents an important parameter in the context of steric stabilization.

\subsection{Size selective precipitation}

The working principle of SSP first reported by the groups of Murray and Weller is as rational as it is simple (Murray C.B. et al., 1993; Vossmeyer T. et al., 1994). Starting from QDs dispersed in a good solvent, a non-solvent is added that induces the preferred, reversible flocculation of larger particles.

In the case of nanoparticles with polar surface termination, a non-solvent would be something non-polar such as toluene or heptane, in the case of nanoparticles with hydrophobic surface properties, a non-solvent would be hydrophilic, e.g. ethanol or acetone. Due to the fact that the flocculates are in the size range of $\mu \mathrm{m}$ to $\mathrm{mm}$, a solid-liquid separation by centrifugation in a typical lab centrifuge with up to $\sim 10000 \mathrm{~g}$ is sufficient to separate the flocculated larger particles from small QDs in the supernatant. As illustrated in Fig. 10, the process can be repeated in several cycles and even redispersion of the QDs on a primary particle level after centrifugation is possible. In the past it was demonstrated in various works that SSP is a highly efficient procedure that is able to substantially narrow PSDs and improve the emission properties of a sample (Komada S. et al., 2012; Nag A. et al., 2007). For instance, Mastronardi et al. subdivided a starting suspension of $\mathrm{Si}$ nanoparticles into the impressive amount of 14 differently sized samples with all of them revealing substantial differences in their optical properties (Mastronardi M.L. et al., 2011).

However, in addition to the final outcome of SSP in terms of samples with small RSD as well as varying absorbance and emission properties, the classification process itself can be analyzed by means of well-known concepts from the field of process engineering and particle technology (Segets D. et al., 2013; Segets D. et al., 2015). This will be briefly summarized in the following section.

Knowing the relative masses of the coarse fraction $g$ $\left(=m_{\text {recovered }} / m_{\text {in }}\right)$ and the fine fraction $f\left(=m_{\text {supernatant }} / m_{\text {in }}\right)$ after drying as well as the PSDs of the feed $q_{\mathrm{F}}(x)$, the coarse $q_{\mathrm{g}}(x)$ and the fines $q_{\mathrm{f}}(x)$ from UV/Vis data, the following parameters are accessible (Segets D. et al., 2015):

(i) The cut size $x_{\mathrm{t}}$ at the intersection between the mass weighted density distribution of the coarse $g \cdot q_{\mathrm{g}}(x)$ and the fines $f \cdot q_{\mathrm{f}}(x)$.

(ii) The separation efficiency:

$$
T(x)=\frac{g q_{\mathrm{g}}(x)}{q_{\mathrm{F}}(x)}
$$

(iii) The separation sharpness $\kappa$ to characterize the steepness of the cut with $x_{25, \mathrm{t}}$ and $x_{75, \mathrm{t}}$ being the particle sizes at which $T(x)$ is 0.25 or 0.75 , respectively:

$$
\kappa=\frac{x_{25, \mathrm{t}}}{x_{75, \mathrm{t}}}
$$

(iv) The yields for coarse $\eta_{\mathrm{g}}$ and fines $\eta_{\mathrm{f}}$ to evaluate the efficiency of the process: 


$$
\eta_{\mathrm{f}}=\frac{\int_{x_{\min }}^{x_{\mathrm{t}}}\left[f q_{\mathrm{f}}(x) \mathrm{d} x\right]}{\int_{x_{\min }}^{x_{\mathrm{f}}}\left[q_{\mathrm{F}}(x) \mathrm{d} x\right]}
$$

For more details the reader is referred to the literature (Leschonski K., 1977; Leschonski K. et al., 1974; Rhodes M.J., 1999; Rumpf H., 1990), however, two important aspects shall be stressed here: the intrinsic inclusion of the mass balance in $T(x)$ (note that $f+g=1$ and $\left.f \cdot q_{\mathrm{f}}(x)+g \cdot q_{\mathrm{g}}(x)=1\right)$ and its independency of the quantity (number, area, volume/mass) based on which the PSD is derived. Thus, $T(x)$ is a perfect measure for the classification process whereas RSD is a perfect measure for the classification result in terms of the derived product PSD.

Using Eqs. 11-14 and performing classification studies on $\mathrm{ZnS}$ QDs with different feed distributions and various good solvent/poor solvent combinations, Segets et al. found the following analytical description of SSP that can be understood as a law of mass action (Segets D. et al., 2015) with dissociation constant $K_{\text {diss }}$ :

$$
K_{\text {diss }, i}\left(x_{i}\right)=\frac{f q_{\mathrm{f}}\left(x_{i}\right)}{g q_{\mathrm{g}}\left(x_{i}\right)}=\frac{q_{\mathrm{F}}\left(x_{i}\right)-g q_{\mathrm{g}}\left(x_{i}\right)}{g q_{\mathrm{g}}\left(x_{i}\right)}=\frac{1-T\left(x_{i}\right)}{T\left(x_{i}\right)}
$$

Thus, the cut of an SSP experiment neither depends on the feed PSD nor on process parameters such as stirring, time of poor solvent exposure or dosing. Even the solids concentration of the feed does not have any influence as long as it does not clearly exceed a comparatively high, critical upper limit (e.g. $>10 \mathrm{~g} \mathrm{~L}^{-1}$ in the case of $\mathrm{ZnS}$ ). SSP is determined in terms of the steepness and position of the cut by the chemical structure of the good and the poor solvent as well as their volume ratio. Comparing the effect of different solvents, the relative permittivity of the final mixture - which is of major importance for the van der Waals interaction - is not the sole influencing factor on SSP. In fact, the specific chemistry of the applied solvents and their interaction with the stabilizing ligands adsorbed at the QD surface are significant as well. This represents the connection between classification results, solubility parameters and steric stabilization and will be described in the following section.

\subsection{Link to Flory-Huggins interaction parameter}

It was found that the best way of data representation is to plot the QD dispersibility of a specific size fraction in a 3-dimensional Hansen space normalized to the Hamaker constant of the core material in the solvent mixture
(Segets D. et al., 2015). Further, it turned out that the HSP of decorated nanoparticles which are understood as the ensemble of QD core plus ligand shell need to be clearly distinguished from the HSP of the unbound, free ligands on a molecular level. The balance of van der Waals attraction and HSP is in agreement with works of the groups of Korgel and Roberts. They reported that in the case of SSP, a steric repulsive force comprised of an elastic and an osmotic interaction due to solvation of the ligand tail by the solvent - the latter being related to solubility parameters-needs to be balanced vs. van der Waals adhesion (Kitchens C.L. et al., 2003; Saunders S.R. and Roberts C.B., 2009; Shah P.S. et al., 2002a; Shah P.S. et al., 2002b; Vincent B. et al., 1986). As recently pointed out by Mori (Mori Y., 2015), this can be extended by electrostatic and Born repulsion, possibly entropic effects such as depletion might need to be considered as well (Mao Y. et al., 1995). However, in line with the findings of Segets et al. (Segets D. et al., 2015), especially the study of Mori revealed that van der Waals adhesion and osmotic interaction are the main contributors to the total interaction.

Analyzing the osmotic term in more detail, two quantities are decisive: the volume fraction of the ligand $\phi$ and the Flory-Huggins interaction parameter $\chi_{12}$ (with index 1 referring to the solvent and index 2 referring to the ligand). Whereas the former can be estimated by the surface coverage or is directly accessible via AUC or small-angle neutron scattering (SANS) (Whitell G.V. and Kitchens C.L., 2010), the latter is even more challenging. It relates to the Hildebrand solubility parameter $\delta_{i}$ (or the cohesive energy density $c_{i}=\delta_{i}{ }^{2}$ ):

$$
\chi_{12}=\frac{V_{\mathrm{m}}}{R T}\left(\delta_{1}-\delta_{2}\right)^{2}
$$

With $V_{\mathrm{m}}$ being the molar volume, $R$ being the ideal gas constant and $T$ being the temperature.

Hansen subdivided the energy of cohesion into a polar contribution $\delta_{\mathrm{p}}$, a disperse contribution $\delta_{\mathrm{d}}$ and the ability to exchange electrons $\delta_{\mathrm{h}}$ according to the concept of Lewis acids and bases (Hansen C.M., Hansen C.M. and Skaarup K.J., 1967). Eq. 16 can be rewritten as (Hansen C.M., 2007):

$$
\begin{aligned}
\chi_{12}= & \frac{V_{\mathrm{m}}}{R T}\left[\left(\delta_{\mathrm{d} 2}-\delta_{\mathrm{d} 1}\right)^{2}+0.25\left(\delta_{\mathrm{p} 2}-\delta_{\mathrm{p} 1}\right)^{2}\right. \\
& \left.+0.25\left(\delta_{\mathrm{h} 2}-\delta_{\mathrm{h} 1}\right)^{2}\right]
\end{aligned}
$$

Thus, once having access to the HSP of small nanoparticles, $\chi_{12}$ can be derived. Together with additional knowledge of the surface coverage, the osmotic interaction potential is accessible and steric effects can be balanced vs. van der Waals adhesion.

By the careful analysis of PSDs established from absorbance spectra, the principal applicability of HSP to QDs 
gould, vêready be demonstrated (Segets D. et al., 2015). Now suitable measurement techniques for HSP, for instance by using analytical centrifugation in the liquid phase (Lerche D. and Sobisch T., 2014), need to be established and validated against numeric data like it was already done in the case of polymers (Díaz I. et al., 2013) or gas-phase results, e.g. obtained by inverse gas chromatography (Liu Y. and Shi B., 2008). Thus, the careful evaluation of QD dispersity not only gives important insights into the classification process itself but can even make an important contribution to a better understanding of the steric stabilization of small colloids.

\section{Conclusion and perspectives}

Different methods for analyzing the particle size distribution (PSD) of quantum dots (QD) were discussed. In addition to standard techniques such as high-resolution (HR) transmission electron microscopy (TEM) and dynamic light scattering (DLS) as well as advanced techniques such as small-angle X-ray scattering (SAXS), Field-Flow Fractionation (FFF) and analytical ultracentrifugation (AUC), the focus was put on the conversion of absorbance spectra to PSDs. In this context four approaches were presented, with two of them (according to Mićić et al. and Viswanatha et al.) using predefined PSD shapes (lognormal and Gaussian) and two of them (according to Pesika et al. and Segets et al.) using a step-wise deconvolution approach. The two latter are seen to be preferred whenever the shape of the target PSD is not known. The technique of Pesika et al. uses the local slope of the absorbance spectrum. The approach of Segets et al. uses a shifted bulk absorption coefficient that brings the possibility to include the optical properties of discrete QD size fractions as soon as they are available. Worthy of note is that data of such monodisperse fractions is becoming more and more available, e.g. by using AUC equipped with multiwavelength (MWL) detection.

In the last part of this work, a clear perspective of exactly why precise PSD characterization on an Ångström level matters was presented. A colloidal classification technique that is widely applied in the field of QDs, the so-called size selective precipitation (SSP) was introduced. It is based on the precise tailoring of steric interactions vs. van der Waals adhesion and can be evaluated with established routines from the field of process engineering and particle technology. SSP is not only described well by the separation efficiency $T(x)$ following a law of mass action, but classification results can be illustrated in a 3D Hansen space normalized to van der Waals attraction.

Based on this finding, knowledge on PSDs potentially allows the determination of Hansen solubility parameters (HSP). The latter give access to the osmotic interaction potential originating from the ligand shells adsorbed at the QD surface and thus lead to a better description of steric stabilization. This is of major importance not only with respect to tailored classification processes but also with respect to formulation issues.

\section{Acknowledgments}

The support of the Deutsche Forschungsgemeinschaft (DFG) is gratefully acknowledged through the Cluster of Excellence "Engineering of Advanced Materials". Moreover, I want to thank Johannes Walter and Wolfgang Peukert for fruitful discussion.

\section{References}

Abécassis B., Bouet C., Garnero C., Constantin D., Lequeux N., Ithurria S., Dubertret B., Pauw B.R., Pontoni D., Real-time in situ probing of high-temperature quantum dots solution synthesis, Nano Lett., 15 (2015) 2620-2626.

Alivisatos A.P., Semiconductor clusters, nanocrystals, and quantum dots, Science, 271 (1996) 933-937.

Alvarado S.R., Guo Y., Purnima T., Ruberu P.A., Tavasoli E., Vela J., Inorganic chemistry solutions to semiconductor nanocrystal problems, Coordin. Chem. Rev., 263-264 (2014) 182-196.

Baalousha M., Stolpe B., Lead J.R., Flow field-flow fractionation for the analysis and characterization of natural colloids and manufactured nanoparticles in environmental systems: A critical review, J. Chromatogr. A, 1218 (2011) 4078-4103.

Balbo A., Minor K.H., Velikovsky C.A., Mariuzza R.A., Peterson C.B., Schuck P., Studying multiprotein complexes by multisignal sedimentation velocity analytical ultracentrifugation, Proc. Natl. Acad. Sci. U.S.A., 102 (2005) 81-86.

Bergström L., Meurk A., Arwin H., Rowcliffe D.J., Estimation of Hamaker constants of ceramic materials from optical data using Lifshitz theory, J. Am. Ceram. Soc., 79 (1996) 339-348.

Bhattacharyya S.K., Maciejewska P., Börger L., Stadler M., Gülsün A.M., Cicek H.B., Cölfen H., Development of a fast fiber based UV-Vis multiwavelength detector for an ultracentrifuge, Prog. Coll. Pol. Sci. S, 131 (2006) 9-22.

Borrelli N.F., Smith D.W., Quantum confinement of PbS microcrystals in glass, J. Non.-Cryst. Solids, 180 (1994) 25-31.

Brookes E.H., Demeler B., Monte Carlo analysis of sedimentation experiments, Colloid Polym. Sci., 286 (2008) 138-148.

Brus L., Electronic wave functions in semiconductor clusters: Experiment and theory, J. Phys. Chem., 90 (1986) 25552560.

Brus L.E., A simple model for the ionization potential, electron affinity, and aqueous redox potentials of small semiconductor crystallites, J. Chem. Phys., 79 (1983) 5566-5571.

Brus L.E., Electron-electron and electron-hole interactions in small semiconductor crystallites: The size dependence of 
ef an ar.

thetrowest exc

Cademartiri L., Montanari E., Calestani G., Migliori A., Guagliardi A., Ozin G.A., Size-dependent extinction coefficient of $\mathrm{PbS}$ quantum dots, J. Am. Chem. Soc., 128 (2006) 10337-10346.

Caetano B.L., Santilli C.V., Meneau F., Briois V., Pulcinelli S.H., In situ and simultaneous UV-Vis/SAXS and UV-Vis/XAFS time-resolved monitoring of $\mathrm{ZnO}$ quantum dots formation and growth, J. Phys. Chem. C, 115 (2010) 4404-4412.

Carney R.P., Kim J.K., Qian H., Jin R., Mehenni H., Stellacci F., Bakr O.M., Determination of nanoparticle size distribution together with density or molecular weight by 2D analytical ultracentrifugation, Nat. Commun., 2 (2011) 1-8.

Choi J.-H., Fafarman A.T., Oh S.J., Ko D.-K., Kim D.K., Diroll B.T., Muramoto S., Gillen J.G., Murray C.B., Kagan C.R., Bandlike transport in strongly coupled and doped quantum dot solids: A route to high-performance thin-film electronics, Nano Lett., 12 (2012) 2631-2638.

Cottingham K., Quantum dots leave the light on, Anal. Chem., 77 (2005) 354A-357A.

Dai Q., Wang Y., Li X., Zhang Y., Pellegrino D.J., Zhao M., Zou B., Seo J.T., Wang Y., Yu W.W., Size-dependent composition and molar extinction coefficient of $\mathrm{PbSe}$ semiconductor nanocrystals, ACS Nano, 3 (2009) 1518-1524.

Debnath R., Bakr O.M., Sargent E.H., Solution-processed colloidal quantum dot photovoltaics: A perspective, Energy Environ. Sci., 4 (2011) 4870-4881.

Detloff T., Lerche D., Centrifugal separation in tube and disc geometries: Experiments and theoretical models, Acta Mech., 201 (2008) 83-94.

Díaz I., Díez E., Camacho J., León S., Ovejero G., Comparison between three predictive methods for the calculation of polymer solubility parameters, Fluid Phase Equilib., 337 (2013) 6-10.

Dieckmann Y., Cölfen H., Hofmann H., Petri-Fink A., Particle size distribution measurements of manganese-doped $\mathrm{ZnS}$ nanoparticles, Anal. Chem., 81 (2009) 3889-3895.

Duonghong D., Ramsden J., Grätzel M., Dynamics of interfacial electron-transfer processes in colloidal semiconductor systems, J. Am. Chem. Soc., 104 (1982) 2977-2985.

Embden J.V., Sader J.E., Davidson M., Mulvaney P., Evolution of colloidal nanocrystals: Theory and modeling of their nucleation and growth, J. Phys. Chem. C, 113 (2009) 16342-16355.

Hagendorfer H., Kaegi R., Parlinska M., Sinnet B., Ludwig C., Ulrich A., Characterization of silver nanoparticle products using asymmetric flow field flow fractionation with a multidetector approach-a comparison to transmission electron microscopy and batch dynamic light scattering, Anal. Chem., 84 (2012) 2678-2685.

Hansen C.M., The official Hansen solubility parameter site, $<$ www.hansen-solubility.com> (accessed Aug 8, 2014).

Hansen C.M., Hansen solubility parameters: A user's handbook, $2^{\text {nd }}$ edition, CRC Press, Boca Raton, FL, USA, 2007.

Hansen C.M., Skaarup K.J., The three dimensional solubility parameter-key to paint component affinities: III independent calculations of the parameter components, J. Paint
Technol., 39 (1967) 511-520.

Jafarzadeh S., Thormann E., Rönnevall T., Adhikari A., Sundell P.-E., Pan J., Claesson P.M., Toward homogeneous nanostructured polyaniline/resin blends, ACS Appl. Mater. Interfaces, 3 (2011) 1681-1691.

Kane R.S., Cohen R.E., Silbey R., Theoretical study of the electronic structure of PbS nanoclusters, J. Phys. Chem., 100 (1996) 7928-7932.

Keshari A.K., Pandey A.C., Size and distribution: A comparison of XRD, SAXS and SANS study of II-VI semiconductor nanocrystals, J. Nanosci. Nanotech., 8 (2008) 1221-1227.

Kitchens C.L., McLeod M.C., Roberts C.B., Solvent effects on the growth and steric stabilization of copper metallic nanoparticles in AOT reverse micelle systems, J. Phys. Chem. B, 107 (2003) 11331-11338.

Komada S., Kobayashi T., Arao Y., Tsuchiya K., Mori Y., Optical properties of manganese-doped zinc sulfide nanoparticles classified by size using poor solvent, Adv. Powder Technol., 23 (2012) 872-877.

Koole R., Allan G., Delerue C., Meijerink A., Vanmaekelbergh D., Houtepen A.J., Optical investigation of quantum confinement in $\mathrm{PbSe}$ nanocrystals at different points in the Brillouin zone, Small, 4 (2008) 127-133.

Kowalczyk B., Lagzi I., Grzybowski B.A., Nanoseparations: Strategies for size and/or shape-selective purification, Curr. Op. Coll. Int. Sci., 16 (2011) 135-148.

Krause B., Mende M., Pötschke P., Petzold G., Dispersability and particle size distribution of CNTs in an aqueous surfactant dispersion as a function of ultrasonic treatment time, Carbon, 48 (2010) 2746-2754.

Kuczinski J., Thomas J.K., Photochemistry at the surface of colloidal cadmium sulfide, Chem. Phys. Lett., 88 (1982) 445-447.

Lees E.E., Gunzburg M.J., Nguyen T.-L., Howlett G.J., Rothacker J., Nice E.C., Clayton A.H.A., Mulvaney P., Experimental determination of quantum dot size distributions, ligand packing densities, and bioconjugation using analytical ultracentrifugation, Nano Lett., 8 (2008) 28832890

Lerche D., Sobisch T., Evaluation of particle interactions by in situ visualization of separation behaviour Coll. Surf. A: Physicochem. Aspects, 440 (2014) 122-130.

Leschonski K., Das Klassieren disperser Feststoffe in gasförmigen Medien, Chem.-Ing.-Tech., 49 (1977) 708-719.

Leschonski K., Alex. W., Koglin B., Teilchengrößenanalyse, Chem.-Ing.-Tech., 46 (1974) 23-26.

Lin W., Walter J., Burger A., Maid H., Hirsch A., Peukert W., Segets D., A general approach to study the thermodynamics of ligand adsorption to colloidal surfaces demonstrated by means of catechols binding to zinc oxide quantum dots, Chem. Mater., 27 (2015) 358-369.

Lipovskii A., Kolobkova E., Petrikov V., Kang I., Olkhovets A., Krauss T., Thomas M., Silcox J., Wise F., Shen Q., Kycia S., Synthesis and characterization of PbSe quantum dots in phosphate glass, Appl. Phys. Lett., 71 (1997) 3406-3408.

Liu Y., Gibbs M., Puthussery J., Gaik S., Ihly R., Hillhouse H.W., Law M., Dependence of carrier mobility on nanocrystal size and ligand length in PbSe nanocrystal solids, 
dat Lett, 10 (2010) 1960-1969.

Liany., Shi B., Determination of Flory interaction parameters between polyimide and organic solvents by HSP theory and IGC, Polym. Bull., 61 (2008) 501-509.

Ma W., Swisher S.L., Ewers T., Engel J., Ferry V.E., Atwater H.A., Alivisatos A.P., Photovoltaic performance of ultrasmall PbSe quantum dots, ACS Nano, 5 (2011) 8140-8147.

Mao Y., Cates M.E., Lekkerkerker H.N.W., Depletion force in colloidal systems, Physica A, 222 (1995) 10-24.

Marczak R., Segets D., Voigt M., Peukert W., Optimum between purification and colloidal stability of $\mathrm{ZnO}$ nanoparticles, Adv. Powder Technol., 21 (2010) 41-49.

Mastronardi M.L., Maier-Flaig F., Faulkner D., Henderson E.J., Kübel C., Lemmer U., Ozin G.A., Size-dependent absolute quantum yields for size-separated colloidally-stable silicon nanocrystals, Nano Lett., 12 (2011) 337-342.

Mićić O.I., Cheong H.M., Fu H., Zunger A., Sprague J.R., Mascarenhas A., Nozik A.J., Size-dependent spectroscopy of InP quantum dots, J. Phys. Chem. B, 101 (1997) 49044912.

Mićić O.I., Curtis C.J., Jones K.M., Sprague J.R., Nozik A.J., Synthesis and characterization of InP quantum dots, J. Phys. Chem., 98 (1994) 4966-4969.

Moreels I., Lambert K., De Muynck D., Vanhaecke F., Poelman D., Martins J.C., Allan G., Hens Z., Composition and size-dependent extinction coefficient of colloidal $\mathrm{PbSe}$ quantum dots, Chem. Mater., 19 (2007) 6101-6106.

Moreels I., Lambert K., Smeets D., De Muynck D., Nollet T., Martins J.C., Vanhaecke F., Vantomme A., Delerue C., Allan G., Hens Z., Size-dependent optical properties of colloidal PbS quantum dots, ACS Nano, 3 (2009) 3023-3030.

Mori Y., Retention behavior of colloidal dispersions in sedimentation field-flow fractionation, Adv. Colloid Interface Sci., 53 (1994) 129-140.

Mori Y., Size-selective separation techniques for nanoparticles in liquid, KONA Powder and Particle Journal, 32 (2015) 102-114.

Moynihan T., What are quantum dots, and why do I want them in my TV? <www.Wired.Com/2015/01/primer-quantum-dot/>, WIRED.com, 2015 (accessed April 8, 2015)

Murray C.B., Norris D.J., Bawendi M.G., Synthesis and characterization of nearly monodisperse $\mathrm{CdE}(\mathrm{E}=\mathrm{S}$, Se, Te) semiconductor nanocrystallites, J. Am. Chem. Soc., 115 (1993) 8706-8715.

Nag A., Sapra S., Nagamani C., Sharma A., Pradhan N., Bhat S.V., Sarma D.D., A study of $\mathrm{Mn}^{2+}$ doping in CdS nanocrystals, Chem. Mater., 19 (2007) 3252-3259.

Nielsen M.H., Li D., Zhang H., Aloni S., Han T.Y.-J., Frandsen C., Seto J., Banfield J.F., Cölfen H., De Yoreo J.J., Investigating processes of nanocrystal formation and transformation via liquid cell TEM, Microsc. Microanal., 20 (2014) 425-436.

Nightingale A.M., de Mello J.C., Microscale synthesis of quantum dots, J. Mater. Chem., 20 (2010) 8454-8463.

Oshima H., Theory of electrostatics and electrokinetics of soft particles, Sci. Technol. Adv. Mater., 10 (2009) 063001.

Palik E.D., Handbook of optical constants of solids, Academic Press, London, 1985.
Park J., Joo J., Kwon S.G., Jang Y., Hyeon T., Synthesis of monodisperse spherical nanocrystals, Angew. Chem. Int. Ed., 46 (2007) 4630-4660.

Pauw B.R., Everything SAXS: Small-angle scattering pattern collection and correction, J. Phys.: Condens. Matter, 25 (2013) 383201

Peng X., Wickham J., Alivisatos A.P., Kinetics of II-VI and IIIV colloidal semiconductor nanocrystal growth: "Focusing" of size distributions, J. Am. Chem. Soc., 120 (1998) 53435344.

Pesika N.S., Stebe K.J., Searson P.C., Determination of the particle size distribution of quantum nanocrystals from absorbance spectra, Adv. Mater., 15 (2003a) 1289-1291.

Pesika N.S., Stebe K.J., Searson P.C., Relationship between absorbance spectra and particle size distributions for quantum-sized nanocrystals, J. Phys. Chem. B, 107 (2003b) 10412-10415.

Pietryga J.M., Schaller R.D., Werder D., Stewart M.H., Klimov V.I., Hollingsworth J.A., Pushing the band gap envelope: Mid-infrared emitting colloidal PbSe quantum dots, J. Am. Chem. Soc., 126 (2004) 11752-11753.

Polte J., Erler R., Thünemann A.F., Sokolov S., Ahner T.T., Rademann K., Emmerling S., Kraehnert R., Nucleation and growth of gold nanoparticles studied via in situ small angle $\mathrm{X}$-ray scattering at millisecond time resolution, ACS Nano, 4 (2010) 1076-1082.

Reindl A., Peukert W., Intrinsically stable dispersions of silicon nanoparticles, J. Coll. Int. Sci., 325 (2008) 173-178.

Rhodes M.J., Introduction to particle technology, John Wiley \& Sons Ltd., London, 1999.

Rogach A.L., Talapin D.V., Shevchenko E.V., Kornowski A., Haase M., Weller H., Organization of matter on different size scales: monodisperse nanocrystals and their superstructures, Adv. Funct. Mater., 12 (2002) 653-664.

Rossetti R., Ellison J.L., Gibson J.M., Brus L.E., Size effects in the excited states of small colloidal CdS crystallites, J. Chem. Phys., 80 (1984) 4464-4469.

Rumpf H., Particle technology, Chapmann and Hall, London, 1990.

Sapsford K.E., Tyner K.M., Dair B.J., Deschamps J.R., Medintz I.L., Analyzing material bioconjugates: A review of current and emerging purification and characterization techniques, Anal. Chem., 83 (2011) 4453-4488.

Sashchiuk A., Langof L., Chaim R., Lifshitz E., Synthesis and characterization of $\mathrm{PbSe}$ and $\mathrm{PbSe} / \mathrm{PbS}$ core-shell colloidal nanocrystals, J. Cryst. Growth, 240 (2001) 431-438.

Saunders S.R., Roberts C.B., Size-selective fractionation of nanoparticles at an application scale using $\mathrm{CO}_{2}$ gas-expanded liquids, Nanotechn., 20 (2009) 475605.

Segets D., Gradl J., Klupp Taylor R., Vassilev V., Peukert W., Analysis of optical absorbance spectra for the determination of $\mathrm{ZnO}$ nanoparticle size distribution, solubility and surface energy, ACS Nano, 3 (2009) 1703-1710.

Segets D., Komada S., Butz B., Spiecker E., Mori Y., Peukert W., Quantitative evaluation of the size selective precipitation of Mn-doped $\mathrm{ZnS}$ quantum dots by size distributions calculated from UV/Vis absorbance spectra, J. Nanopart. Res., 15 (2013) 1486. 
Sdgets Đ., Lucas J.M., Klupp Taylor R.N., Scheele M., Zheng H., WAlivisatos A.P., Peukert W., Determination of the quantum dot bandgap dependence on particle size from optical absorbance and transmission electron microscopical measurements, ACS Nano, 6 (2012) 9021-9032.

Segets D., Lutz C., Yamamoto K., Komada S., Süß S., Mori Y., Peukert W., Classification of zinc sulfide quantum dots by size: Insights into the particle surface-solvent interaction of colloids, J. Phys. Chem. C, 119 (2015) 4009-4022.

Segets D., Peukert W., UV/Vis-spektroskopie zur in-situteilchengrößenanalyse, Laborpraxis, (2014).

Seto J., Ma Y., Davis S.A., Meldrum F., Gourrier A., Kim Y.-Y., Schilde U., Sztucki M., Burghammer M., Maltsev S., Jäger C., Cölfen H., Structure-property relationships of a biological mesocrystal in the adult sea urchin spine, Proc. Natl. Acad. Sci. U.S.A., 109 (2012) 3699-3704.

Shah P.S., Holmes J.D., Johnston K.P., Korgel B.A., Size-selective dispersion of dodecanethiol-coated nanocrystals in liquid and supercritical ethane by density tuning, J. Phys. Chem. B, 106 (2002a) 2545-2551.

Shah P.S., Husain S., Johnston K.P., Korgel B.A., Role of steric stabilization on the arrested growth of silver nanocrystals in supercritical carbon dioxide, J. Phys. Chem. B, 106 (2002b) 12178-12185.

Steckel J.S., Coe-Sullivan S., Bulovic V., Bawendi M.G., $1.3 \mu \mathrm{m}$ to $1.55 \mu \mathrm{m}$ tunable electroluminescence from $\mathrm{PbSe}$ quantum dots embedded within an organic device, Adv. Mater., 15 (2003) 1862-1866.

Steigerwald M.L., Brus L.E., Semiconductor crystallites: A class of large molecules, Acc. Chem. Res., 23 (1990) 183188.

Strauss H.M., Karabudak E., Bhattacharyya S., Kretzschmar A., Wohlleben W., Cölfen H., Performance of a fast fiber based UV/Vis multiwavelength detector for the analytical ultracentrifuge, Colloid Polym. Sci., 286 (2008) 121-128.

Sun Y., Ren Y., In situ synchrotron X-ray techniques for realtime probing of colloidal nanoparticle synthesis, Part. Part. Sys. Charact., 30 (2013) 399-419.

Sun Y., Welch G.C., Leong W.L., Takacs C.J., Bazan G.C., Heeger A.J., Solution-processed small-molecular solar cells with $6.7 \%$ efficiency, Nature Mater., 11 (2012) 44-48.

Tyrakowski C.M., Snee P.T., A primer on the synthesis, water-solubilization, and functionalization of quantum dots, their use as biological sensing agents, and present status, Phys. Chem. Chem. Phys., 16 (2014) 837-855.

Vincent B., Edwards J., Emmett S., Jones A., Depletion flocculation in dispersions of sterically-stabilised particles ("soft spheres"), Colloid Surface, 18 (1986) 261-281.

Viswanatha R., Sapra S., Satpati B., Satyam P.V., Dev B.N., Sarma D.D., Understanding the quantum size effects in ZnO nanocrystals, J. Mater. Chem., 14 (2004) 661-668.

Viswanatha R., Sarma D.D., Study of the growth of capped $\mathrm{ZnO}$ nanocrystals: A route to rational synthesis, Chem. Eur. J., 12 (2006) 180-186.

Vossmeyer T., Katsikas L., Giersig M., Popovic I.G., Diesner K., Chemseddine A., Eychmüller A., Weller H., CdS nanoclusters: Synthesis, characterization, size dependent oscillator strength, temperature shift of the excitonic transition energy, and reversible absorbance shift, J. Phys. Chem., 98 (1994) 7665-7673.

Walter J., Löhr K., Karabudak E., Reis W., Mikhael J., Peukert W., Wohlleben W., Cölfen H., Multidimensional analysis of nanoparticles with highly disperse properties using multiwavelength analytical ultracentrifugation, ACS Nano, 8 (2014) 8871-8886.

Walter J., Nacken T.J., Thajudeen T., Eigler S., Peukert W., Determination of the lateral dimension of graphene oxide nanosheets using analytical ultracentrifugation, Small, 11 (2015a) 814-825.

Walter J., Sherwood P.J., Lin W., Segets D., Stafford W.F., Peukert W., Simultaneous analysis of hydrodynamic and optical properties using analytical ultracentrifugation equipped with multiwavelength detection, Anal. Chem., 87 (2015b) 3396-3403.

Walter J., Thajudeen T., Süß S., Segets D., Peukert W., New possibilities of accurate particle characterisation by applying direct boundary models to analytical centrifugation, Nanoscale, 7 (2015c) 6574-6587.

Whitell G.V., Kitchens C.L., Small-angle neutron scattering of silver nanoparticles in gas-expanded hexane, J. Phys. Chem. C, 114 (2010) 16285-16291.

Williams S.K.R., Runyon J.R., Ashames A.A., Field-flow fractionation: Addressing the nano challenge, Anal. Chem., 83 (2011) 634-642.

Yen B.K., Günther A., Schmidt M.A., Jensen K.F., Bawendi M.G., A microfabricated gas-liquid segmented flow reactor for high-temperature synthesis: The case of CdSe quantum dots, Angew. Chem. Int. Ed., 44 (2005) 5447-5451.

Yoshikawa H., Adachi S., Optical constants of ZnO, Jpn. J. Appl. Phys., 36 (1997) 6237-6243.

Yu W.W., Qu L., Guo W., Peng X., Experimental determination of the extinction coefficient of CdTe, CdSe, and CdS nanocrystals, Chem. Mater., 15 (2003) 2854-2860.

Zheng H., Smith R.K., Jun Y.-W., Kisielowski C., Dahmen U., Alivisatos A.P., Observation of single colloidal platinum nanocrystal growth trajectories, Science, 324 (2009) 13091311.

\section{Nomenclature}

$\begin{array}{ll}\text { Abbreviations } \\ \text { AC } & \text { analytical centrifugation } \\ \text { AsFlFFF } & \text { asymmetric flow field-flow fractionation } \\ \text { AUC } & \text { analytical ultracentrifugation } \\ \text { A4F } & \text { asymmetric flow field-flow fractionation } \\ \text { DLS } & \text { dynamic light scattering } \\ \text { EMA } & \text { effective mass approximation } \\ \text { FFF } & \text { field-flow fractionation } \\ \text { FLN } & \text { fluorescence line narrowing } \\ \text { HR } & \text { high resolution } \\ \text { HSP } & \text { Hansen solubility parameters }\end{array}$


OMP

$\mathrm{PL}^{\mathrm{N}}$

PSD

photoluminescence

QD

particle size distribution

RSD

relative standard deviation

SANS small angle neutron scattering

SAXS small angle X-ray scattering

SSP size selective precipitation

TEM transmission electron microscopy

\section{Greek Symbols}

$\alpha \quad$ absorption coefficient $\left(\mathrm{M}^{-1} \mathrm{~m}^{-1}\right)$

$\alpha_{\text {shift }, i}(\lambda) \quad$ shifted absorption coefficient $\left(\mathrm{M}^{-1} \mathrm{~m}^{-1}\right)$

$\chi \quad$ Flory Huggins parameter $(-)$

$\Delta D_{\text {actual }} \quad$ true width of PSD (m)

$\Delta d_{\text {app }} \quad$ apparent width of absorbance data on a particle size scale $(\mathrm{m})$

$\delta_{\mathrm{d}} \quad$ disperse contribution to HSP $\left(\left(\mathrm{J} \mathrm{m}^{-3}\right)^{0.5}\right)$

$\delta_{\mathrm{h}} \quad$ HSP for ability to exchange electrons $\left(\left(\mathrm{J} \mathrm{m}^{-3}\right)^{0.5}\right)$

$\delta_{\mathrm{i}} \quad$ Hildebrand solubility parameter $\left(\left(\mathrm{J} \mathrm{m}^{-3}\right)^{0.5}\right)$

$\delta_{\mathrm{p}} \quad$ polar contribution to $\operatorname{HSP}\left(\left(\mathrm{J} \mathrm{m}^{-3}\right)^{0.5}\right)$

$\phi \quad$ volume fraction of ligand (-)

$\sigma \quad$ standard deviation (m)

$\eta \quad$ dynamic viscosity (Pa s)

$\eta_{\mathrm{f}} \quad$ yield of fines (-)

$\eta_{\mathrm{g}} \quad$ yield of coarse (-)

$\kappa \quad$ separation sharpness (-)

$\lambda \quad$ wavelength (m)

\section{Latin Symbols}

A

$A(\lambda) \quad$ measured absorbance (-)

$A_{i}{ }^{\prime}(\lambda) \quad$ partial absorbance (-)

a empiric parameter (m)

$b \quad$ empiric parameter (-)

c empiric parameter (m)

C constant $\left(\mathrm{eV}^{0.5} \mathrm{~m}^{-1}\right)$

$\mathrm{c}_{\mathrm{i}} \quad$ cohesive energy density $\left(\mathrm{J} \mathrm{m}^{-3}\right)$

D diffusion coefficient $\left(\mathrm{m}^{2} \mathrm{~s}^{-1}\right)$

$d_{\mathrm{c}} \quad$ optical path length (m)

$E_{\mathrm{g}} \quad$ band gap energy $(\mathrm{eV})$

$f \quad$ relative mass of fine fraction (-)

$g \quad$ relative mass of coarse fraction (-)

$h v \quad$ energy $(\mathrm{J})$

$K_{\text {diss }} \quad$ dissociation constant (-)

$m \quad$ mass $(\mathrm{kg})$

$n$

$n(\mathrm{x})$

$p$

$q_{3}$

$R$

$r$

$T$

$T(x)$

$V_{\mathrm{m}}$

$x$

$x_{\mathrm{h}}$

$x_{\mathrm{t}}$ fitting parameter $(-)$

relative abundance $(-)$

fitting parameter $(-)$

volume density $\left(\mathrm{m}^{-1}\right)$

ideal gas constant $\left(\mathrm{J} \mathrm{mol}^{-1} \mathrm{~K}^{-1}\right)$

particle radius $(\mathrm{m})$

temperature $(\mathrm{K})$

separation efficiency $(-)$

molar volume $\left(\mathrm{m}^{3} \mathrm{~mol}^{-1}\right)$

particle diameter $(\mathrm{m})$

hydrodynamic particle diameter (m)

cut size (m)

\section{Author's short biography}

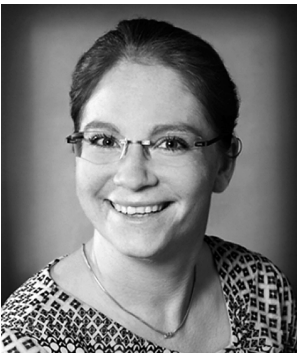

\section{Doris Segets}

Dr. Segets is currently affiliated at the Institute of Particle Technology of the Friedrich-Alexander-Universität Erlangen-Nürnberg (FAU). She received her $\mathrm{PhD}$ in 2013 and her research interests include the characterization and tailoring of technically relevant colloidal interfaces, the development of new concepts for process engineering and the scale-up of colloidal nanoparticles, micro reaction technology and high-throughput experimentation (HTE) as well as the application of well-defined nanoparticles for ultrafiltration and chromatography. 